\title{
A propos d'une autre souche d'Endomyces reessii Van Der Walt 1959
}

\author{
par Henri SAEZ
}

Laboratoire d'Ethologie du Muséum, Parc zoologique, 53, avenue de Saint-Maurice, F. 75 - Paris, $12^{\mathrm{e}}$

\begin{abstract}
Résumé
L'espèce décrite par Van der Walt sous le nom d'Endomyces reessii apparaît comme la forme parfaite de Geotrichum candidum et, comme celui-ci, produit également des endospores. Une seconde souche, reçue sous le nom de Geotrichum suaveolens (Lindner) Ciferri, qui nous a conduit à faire un rappel historique, a aussi été identifiée à $E$. reessi.
\end{abstract}

\section{Summary}

The Van der Walt's species, Endomyces reessii is the perfect form of Geotrichum candidum, and like this fungus produces also endospores. A second species received as Geotrichum suaveolens (Lindner) Ciferri has been identified to E. reessii, and a brief historical discussion was given at this occasion.

Les deux souches étudiées ci-dessous :

- Endomyces reessii NRRL YB-4463, culture reçue du Docteur Wickerham, Peoria,

- Geotrichum suaveolens (Lindner) Ciferri, culture reçue de $\mathrm{M}^{\text {He }}$ le Docteur . Slooff, Delft, dans les conditions que nous expliquerons plus loin,

presentent les mêmes propriétés morpho-physiologiques suivantes : 


\section{I. - Propriétés morphologiques.}

\section{A) Macroscopiques.}

En milieu solide. Sur Sabouraud-glucosé $2 \%$ : bon développement ; la culture ressemble à celle d'un Geotrichum candidum. A température du laboratoire la jeune colonie est blanche, plane ou de section légèrement convexe, duveteuse sur toute sa surface. En vieillissant elle prend une couleur blanc grisâtre ou blanc jaunâtre; s'étale sur le milieu en se plissant quelquefois ; perd souvent son duvet par endroits. La disparition de certains filaments aériens est la conséquence d'une désarticulation plus poussée du thalle.

En milieu liquide. En eau peptonée $1 \%$ et en eau peptonée $1 \%$-glucosée $2 \%$, à $25^{\circ}$ : production précoce (en 24 à 48 heures) d'un voile qui va aller en s'épaississant davantage sur le second milieu où il peut atteindre $1 \mathrm{~cm}$ au bout d'un mois de culture. Véritable colonie développée en surface, ce voile suit également la même évolution ; c'est dire qu'au début les îlots se réunissent rapidement pour former une pellicule blanche et duveteuse, puis celle-ci devient blanc sale, jaunâtre, membraneuse, glabre entièrement ou par zones.

\section{B) Microscopiques.}

A l'examen microscopique on observe aisément la fragmentation du thalle en arthrospores, les endospores, produites par les deux scuches étudiées, doivent être recherchées patiemment.

Au $3^{\circ}$ jour de culture, à $25^{\circ}$, le diamètre des hyphes et le petit et le grand axe des arthrospores mesurent :

\begin{tabular}{|c|c|c|}
\hline & Sur Sabouraud-glucosé & En eau peptonée-glucosée \\
\hline $\begin{array}{l}\text { Mycélium } \ldots \ldots \ldots \ldots \\
\text { Arthrospores }\end{array}$ & (2-7) $\stackrel{2-7}{(4-19,5) \mu}$ & $\stackrel{2-7,5 \mu}{(2-7,5) \stackrel{(4-17,5)}{(} \mu}$ \\
\hline
\end{tabular}

Le mycélium, vrai, hyalin, septé, est constitué de filaments fréquemment ramifiés. Tout comme chez G. candidum, mais moins communément que dans la plupart des souches de cette espèce, les images de bifurcation des sommets des filaments et (ou) de leurs rameaux confèrent au développement du thalle une allure pseudo-dichotomique.

Les arthrospores provenant de la désarticulation totale ou partielle des filaments et (ou) de leurs ramifications abondent dans la jeune colonie. Elles ont une section généralement rectangulaire, rarement carrée ; par la suite leurs angles s'arrondissent, plus rapidement d'ailleurs en milieu liquide qu'en milieu solide, et elles prennent une forme ovale ou sphérique. Une grande proportion d'entre elles germe sitôt leur libération, voire avant que la séparation ne soit achevée. De l'un des angles, ou de l'une des faces, elles émettent un tube (parfois plusieurs) qui, ou bien se fragmente précocément en petits éléments, 


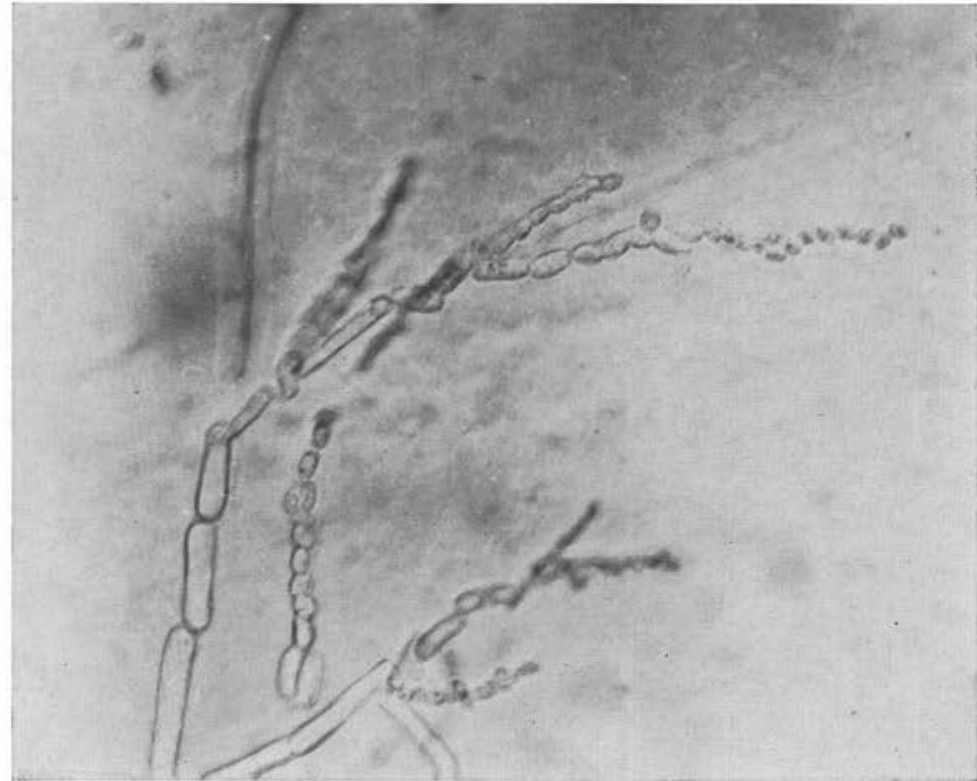

FIG. 1. - Endomyces reessii, souche NRRL. «Lignes brisées »: fragmentation de filaments de différents diamètres. Culture sur Sabouraud glucosé de dix jours à $25^{\circ}$

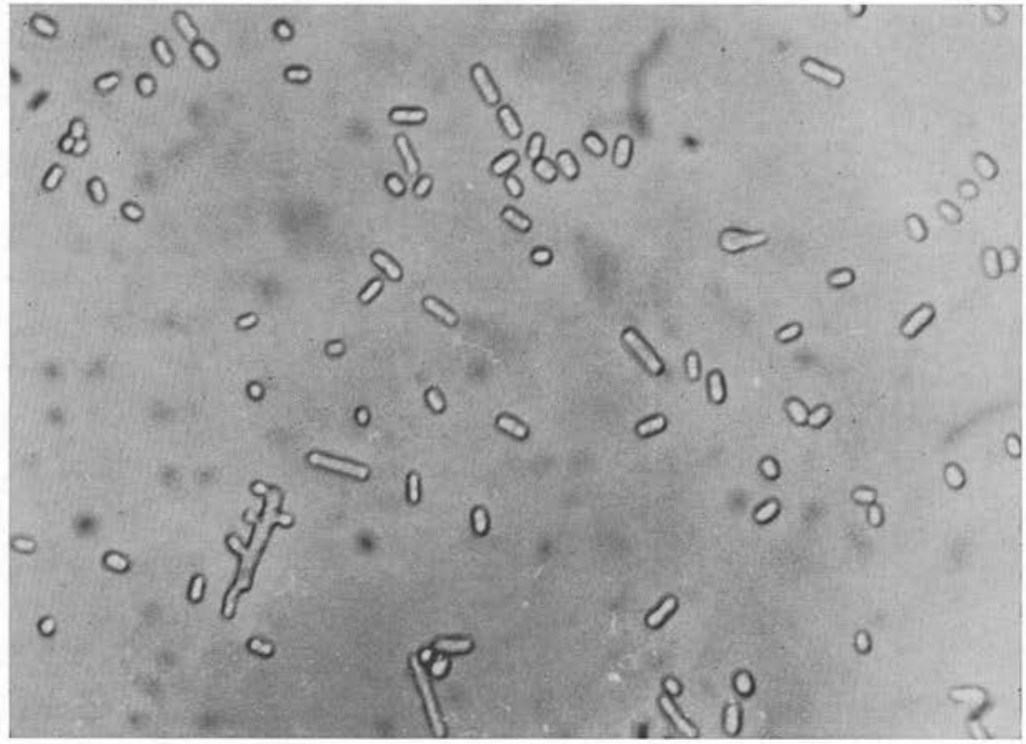

FIG. 2. - Endomyces reessii, souche NRRL, Arthrospores et fragment de filament avec ébauches latérales des formations ascogènes. Culture sur Sabouraud glucosé de trois jours à $25^{\circ}$ 


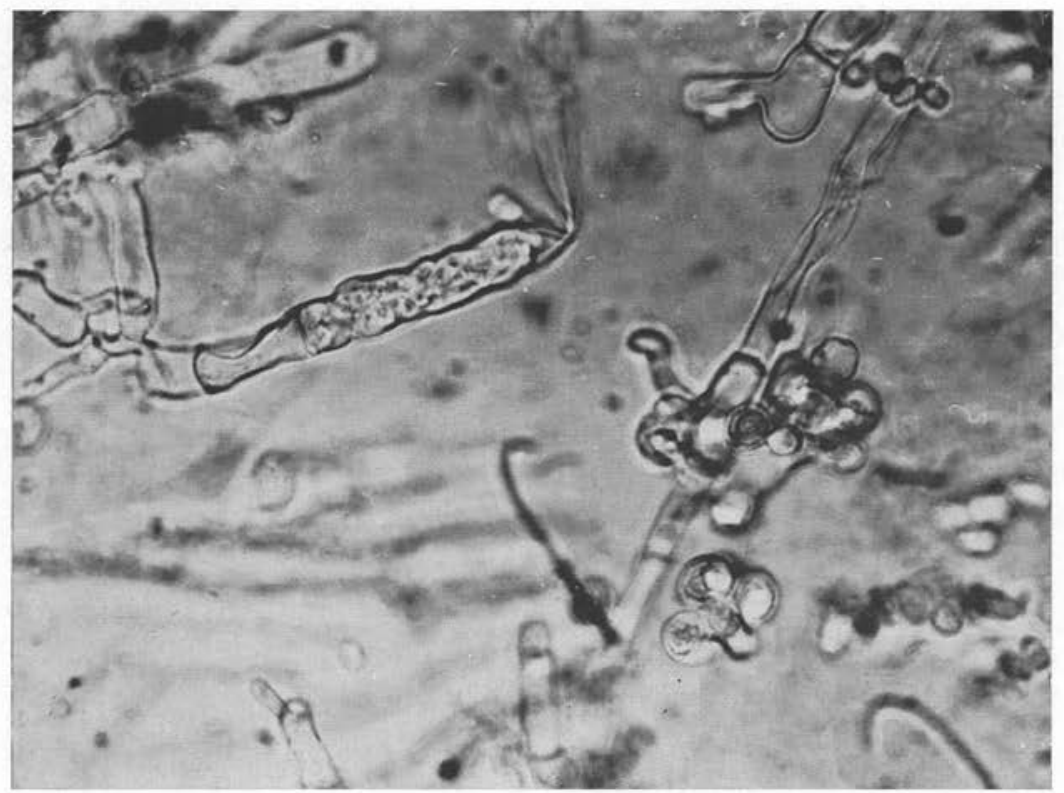

FIG. 3

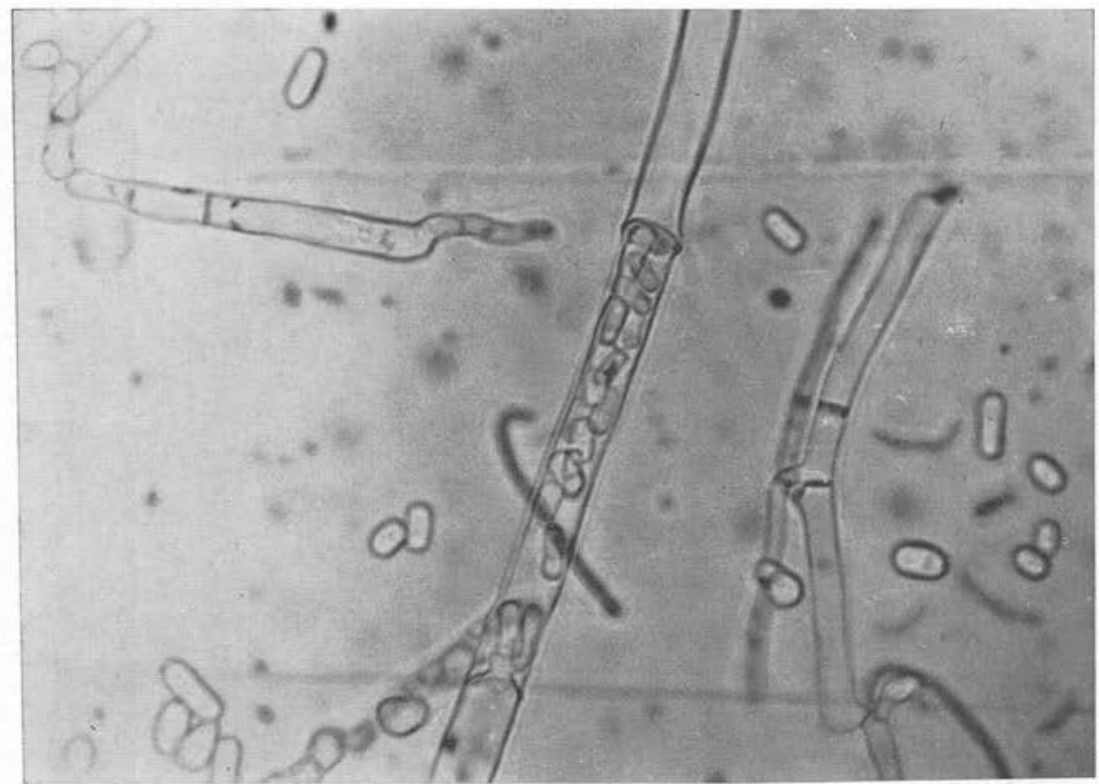

FIG. 4

FIG. 3 et 4. - Endomyces reessii, souche NRRL. Endospores dans un fragment de filament. Cultures sur Sabouraud glucosé, à température du laboratoire, âgées de 31 jours (fig. 3) et 32 jours (fig. 4) 


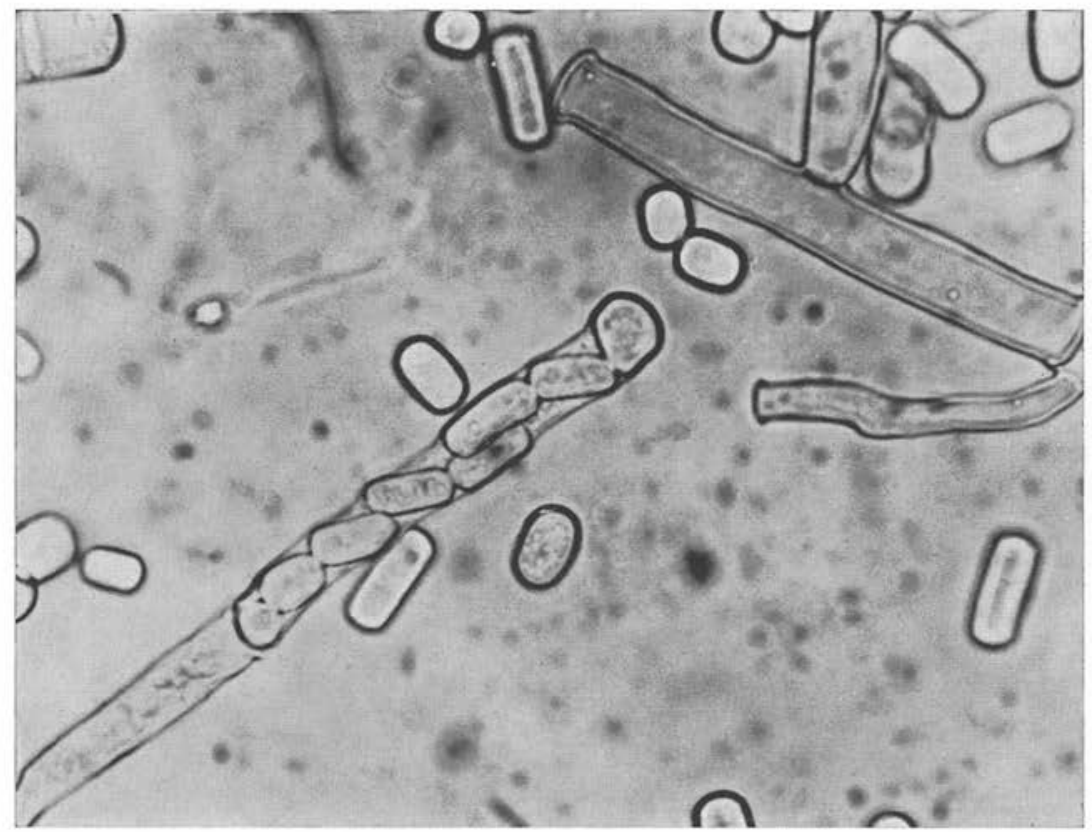

FIG. 5



FIG. 6

FIG. 5 et 6. - Endospores de même type chez: Geotrichum magnum n. comb. (fig. 5), Geotrichum gracile (fig. 6) 


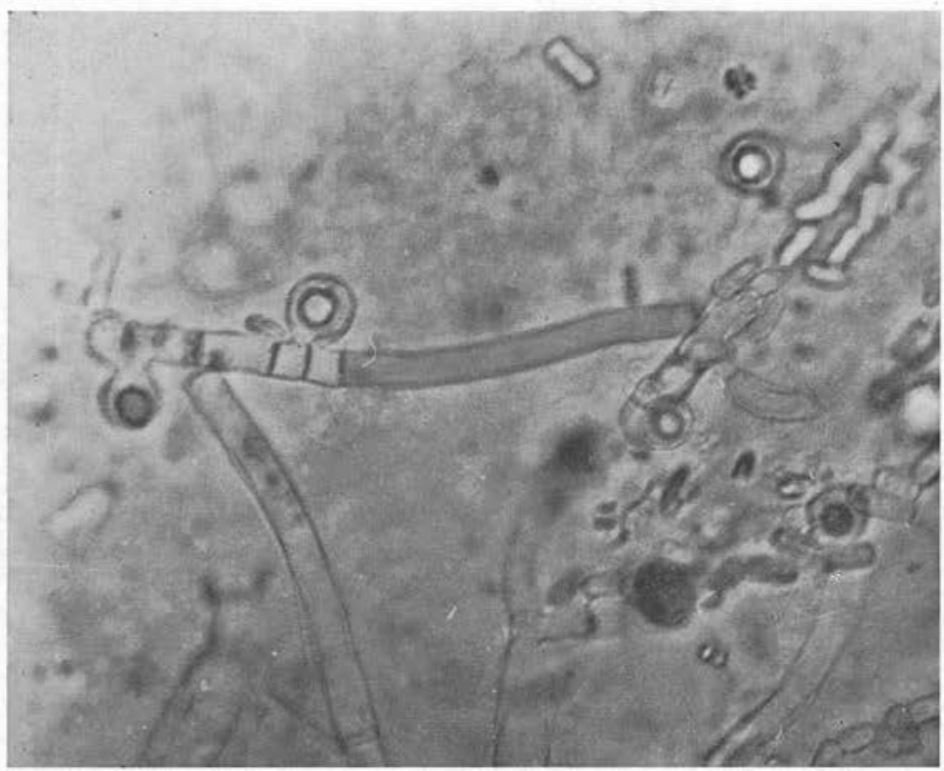

FIg. 7. - E. reessii, souche de Péoria

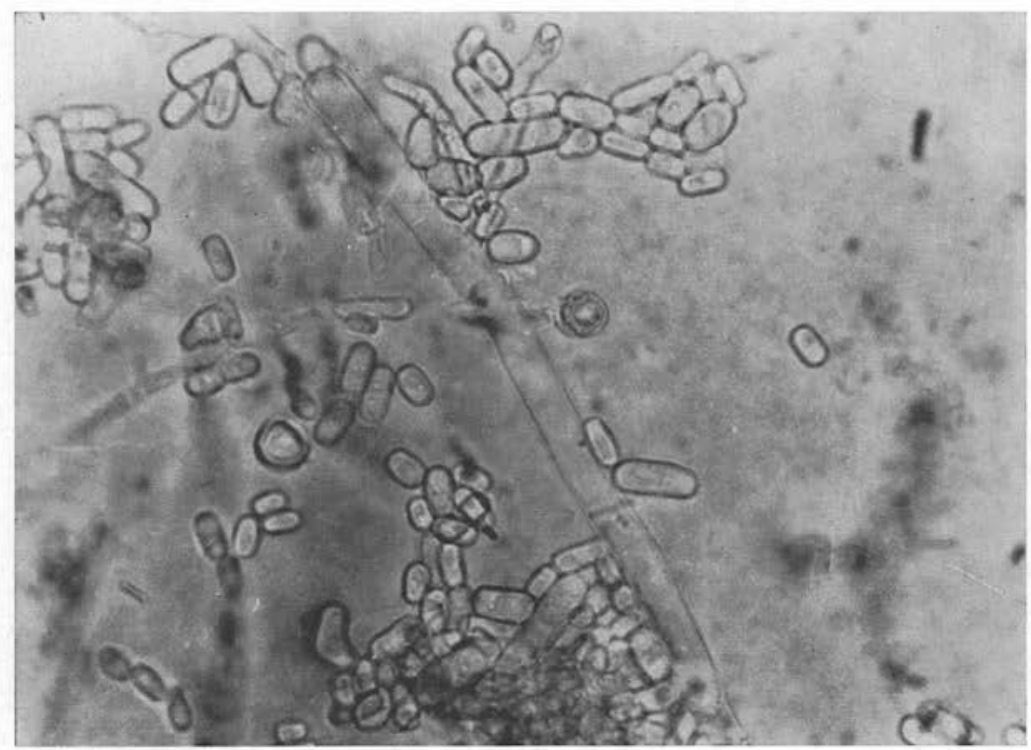

FIG. 8. - Souche de Delft 


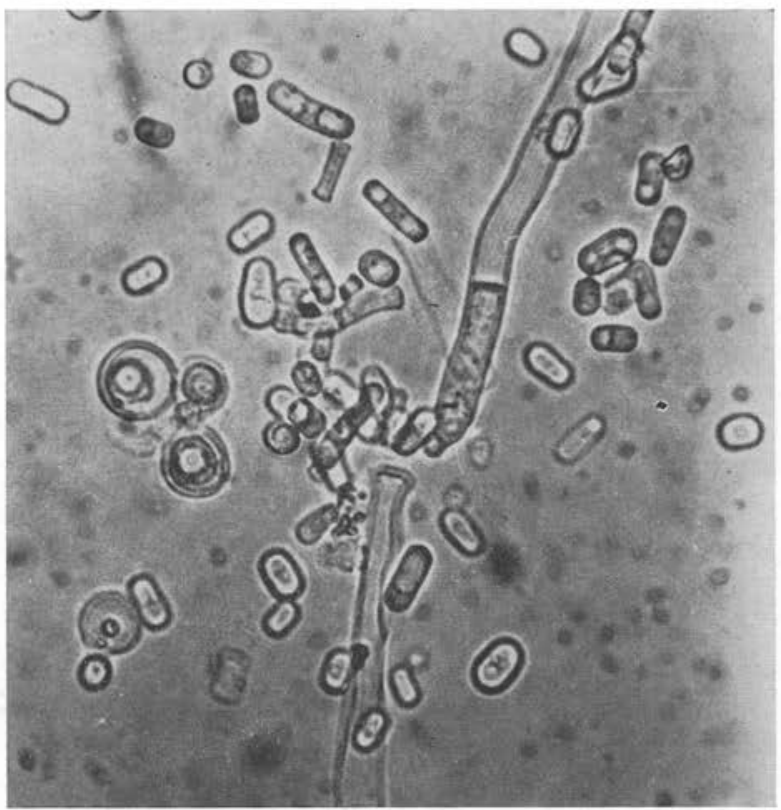

FIG. 9. - Souche de Delft



Fig. 10: Spore germant. Souche de Delft. Culture en eau peptonée. Fig. 7 à 10. - Reproduction sexuée: formations latérales (fig. 7, 8) contre la paroi des hyphes ou au bout de petites protubérances et formations isolées (fig. 9) 
ou au contraire s'allonge, se ramifie et ne se fragmente que tardivement, et le plus souvent partiellement, en de plus grands éléments. Dans les cultures âgées, dont le milieu est en voie d'épuisement, les arthrospores se vacuolisent et demeurent à l'état de repos ; transportées dans un milieu frais, les vacuoles diminuent de volume, le protoplasme se densifie et la plupart d'entre elles recouvrent leur pouvoir de germination (cf. fig. 1 et 2).

Ainsi que chez plusieurs espèces de Geotrichum, (5), (6), (7), nous avons vu des endospores en milieu gélosé (Sabouraud-glucosé, Gorodkowa), dans des colonies de trois à quatre semaines, obtenues à $25^{\circ}$ ou à la température du laboratoire. Elles naissent dans une portion de filament soit apicale soit limitée par deux cloisons. Dans la masse protoplasmique de cette portion apparaissent des septa perpendiculaires au grand axe du filament qui n'atteignent pas la paroi mycélienne externe ; moins habituellement on assiste à un recoupement dans un plan longitudinal, aboutissant à deux rangées parallèles d'endospores. Elles ont la forme des arthrospores mais une taille généralement moindre. Elles se libèrent accidentellement ou par amincissement de la paroi mycélienne externe (cf. fig. 3 à 6).

La reproduction sexuée apparaît très tôt dans les deux souches: sur Sabouraudglucosé, à $30^{\circ}$, nous l'avons surprise dès le quatrième jour de culture. Le phénomène de conjugaison ne semble pas le mode le plus habituel. Les éléments de cette reproduction, isolés ou en grappes, siègent sur les hyphes en position apicale ou latérale, contre la paroi mycélienne ou au bout de petites projections. En fait ils naissent toujours à l'extrémité de protubérances plus ou moins courtes. La partie distale de ces protubérances s'évase en ampoule, le contenu ampullaire devient granuleux, lipidique quelquefois. Nombre de ces formations demeurent à ce stade, dans d'autres se différencient une ou, plus rarement, deux spores rondes de 6-8 $\mu$ de diamètre, parfois légèrement ovales, à membrane épaissie (fig. 7 à 9). Transportées en milieu liquide, en eau peptonée par exemple, certaines spores augmentent quelque peu de volume avant d'émettre un tube germinatif entouré d'une collerette à sa base qui pourrait bien provenir de l'écartement des lèvres d'un pore (fig. 10).

\section{II. - Propriétés physiologiques. (cf. tableau).}

Les deux souches sont azymatiques.

Elles assimilent: précocement, le glucose, le galactose, le xylose, l'alcool et le sorbitol ; faiblement ou tardivement, le mannitol ; faiblement et tardivement, l'adonitol. Nos résultats concordent donc bien avec ceux de Van der Walt (8), exception faite de l'utilisation du mannitol, dont la réaction est cependant faible ou tardive.

Leur température maximale de développement est inférieure à $37^{\circ}$.

\section{Discussion.}

Compte tenu de l'assimilation du mannitol, Endomyces reessii est à considérer comme la forme parfaite de Geotrichum candidum et, comme celui-ci (7), il peut également produire des endospores. 


\section{Tableau}

PROPRIÉTÉS PHYSIOLOGIQUES

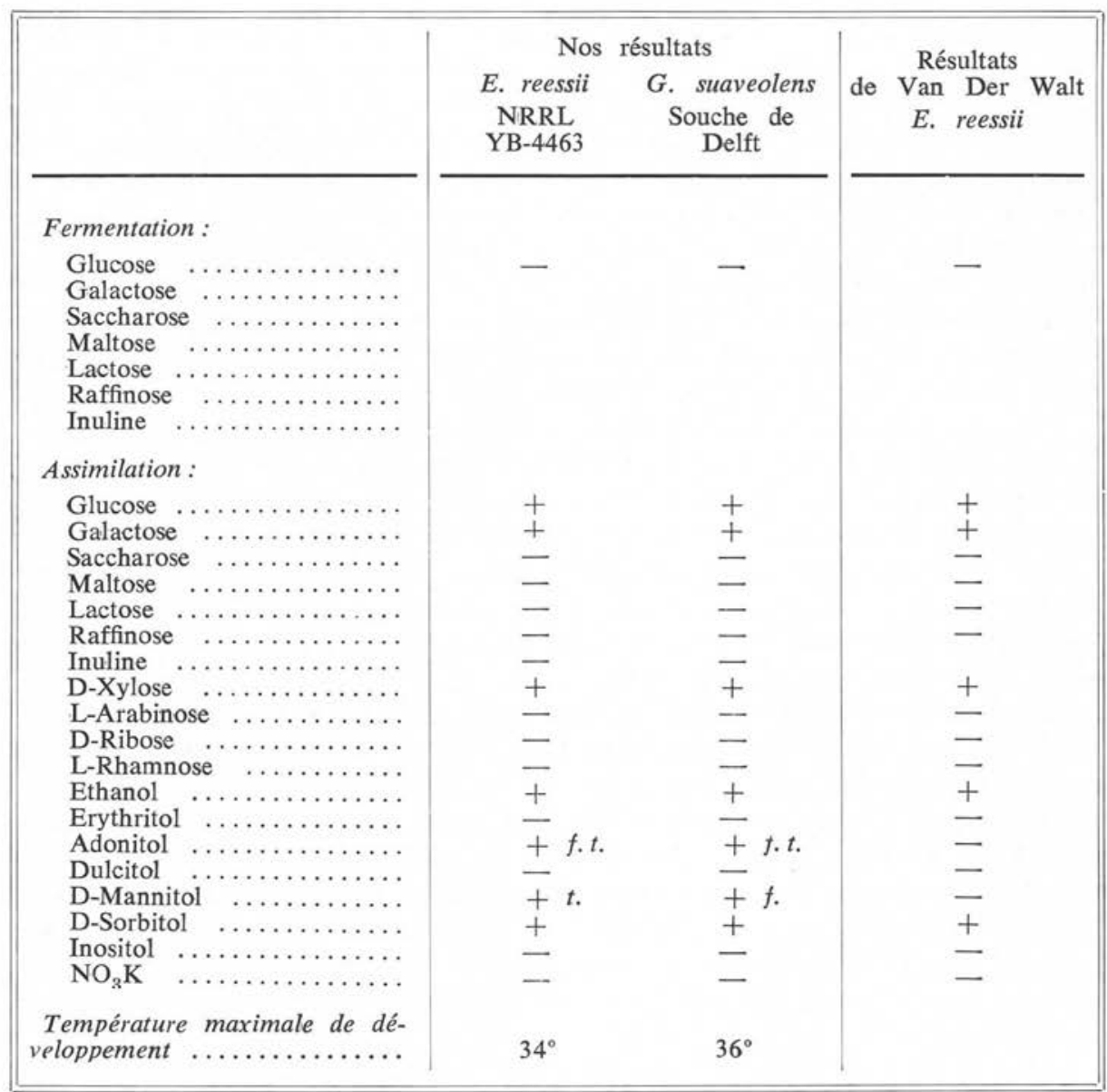

$f .=$ réaction faiblement positive,

$t$. $=$ réaction tardivement positive.

Les propriétés morphologiques et physiologiques de la souche de Delft permettent de l'identifier à $E$. reessii ; la particularité de cette dernière est d'avoir une température maximale de développement $\left(36^{\circ}\right)$ quelque peu supérieure à celle de la souche que nous a envoyée le $\mathrm{P}^{\mathrm{r}}$ Wickerham. Cette différence, de deux degrés centigrades, est minime et entre dans le cadre des variations de l'espèce; rappelons que pour G. candidum nous avons trouvé un intervalle plus grand: $34^{\circ}-39^{\circ}$. 
La deuxième souche étudiée nous intéresse à un autre titre. Elle nous conduit à citer des espèces fongiques dont nous traiterons brièvement d'un historique qui est détaillé dans les publications de Caretta (1), Diddens \& Lodder (2), Lodder \& KregerVan Rij (3), Morenz (4) et Windisch (9).

Lindner a décrit, sous le nom de Sachsia suaveolens, une espèce que Lindau a transféré dans le genre Oospora. Bien après, en 1913, Krzemecki a appelé Oïdium suaveolens un champignon à odeur fruitée. Au laboratoire de Parasitologie de Paris existe une traduction française de la thèse de Berkhout que Langeron avait obtenue du $\mathrm{D}^{r}$ Baudet. Dans ce travail nous lisons: «L'Ö̈dium suaveolens ne pourra être nommé Oospora suaveolens parce que Sachsia suaveolens a été ainsi nommé par Lindau. C'est pour cette raison que le nom de Oospora fragans se rapporte le mieux à l'ancien nom. » Berkhout écrit ceci au chapitre des Recherches personnelles, à propos de l'espèce de Krzemecki, qu'elle juge donc différente de celle de Lindner, et le changement de nom de l'espèce la plus récemment décrite s'explique par l'appartenance des deux champignons au genre Oospora tel qu'on l'entendait alors.

Le champignon de Krzemecki est devenu Endomyces lactis (Fresen.) Windish var. fragans (Berkhout) Morenz 1960. Le $\mathrm{P}^{r}$ Windish nous a fait parvenir la souche $\mathrm{n}^{\circ} 9 \mathrm{~d}^{\prime} E$. lactis var. fragans. De celle-ci nous dirons simplement ici qu'elle fermente bien le glucose, d'une fermentation qui n'a rien à voir avec les cuelques bulles de gaz produites avec ce sucre par certaines souches de G. candidum, et qu'elle n'assimile pas le xylose. De Baarn nous avons obtenu un Cladosporium suaveolens (Lindner) Delitsch : c'est un champignon filamenteux, dont la colonie noircit quelque peu en prenant de l'âge, qui ne peut être classé dans les Geotrichum.

D'un autre côté Cifferi a isolé en République Dominicaine une souche qu'il a envoyée au Centralbureau voor Schimmelcultures (C. B. S.) en 1931 sous le nom de Geotrichum suaveolens (Lindner) Cif. dont font état Diddens \& Lodder (2) et Lodder \& Kreger-Van Rij (3). Dans la monographie des Levures de 1952 il est dit, en substance, que Ciferri fut plus tard d'accord avec le C. B. S. pour penser que cette culture n'est pas identique avec Sachsia suaveolens Lindner, mais qu'elle appartient au genre Geotrichum - «Ciferri pensa alors qu'elle était identique avec Ö̈dium suaveolens Krz. Quoique le C.B.S. ne fut pas d'accord avec l'une ou l'autre de ces identifications il fut admis que la culture de Ciferri était plus proche d'Ödium suaveolens que de Sachsia suaveolens. »

Par parenthèses, la souche de Ciferri a été l'objet d'un malentendu avec Langeron que les Auteurs hollandais ont mis, bien sagement sans doute, sur le compte d'une comtamination. Langeron l'obtint du C. B.S. en 1932, et cn 1938 Langeron \& Guerra en firent une levure dénommée Candida suaveolens (Lindner) Ciferri, lequel fut à son tour mis en synonymie avec Candida humicola (Daszewska) Diddens et Lodder.

Caretta (1) a fait l'étude morphologique de la souche $\mathrm{n}^{\circ} 9$ d'E. lactis var. fragans de Windisch, celle-là même que Morenz et nous avons eue en mains. Il estime que ce champignon est le même que celui isolé par Ciferri, et revalide le nom spécifique : Georichum suaveolens (Lind.) Cif.

Aussi avons-nous essayé de nous procurer la souche de Ciferri. $\mathbf{M}^{\text {11e }}$ Sloof a eu l'extrême obligeance de faire des recherches à ce sujet et de nous communiquer les ren- 
seignements suivants : Geotrichum suaveolens (Lindner) Ciferri fut retiré de la Collection en 1956, à Baarn, et ne figure plus dans la liste des cultures après 1961. Dans une armoire où sont rangées les souches mises de côté elle a découvert une culture portant ce nom qu'elle nous a fait parvenir. Par conséquent, pour autant que le champignon que nous avons étudié soit bien celui isolé par Ciferri, il appartient à l'espèce Endomyces reessii et Geotrichum suaveolens ne pourrait être qu'une nouvelle combinaison valable pour Ö̈dium suaveolens, si Caretta ne faisait suivre cette dénomination de «(Lindner) Cif. ». Mais avec Caretta nous nous étonnons du remplacement du nom de Krzemecki par celui de Berkhout et de la persistance du terme de «fragans » dans le transfert aux genres Endomyces (9), puis Geotrichum (4) de l'espèce : Ö̈dium suaveolens Krzemecki 1913, cités en tête des synonymes des nouvelles combinaisons.

\section{Bibliographie}

1. Caretta (G.), 1960. - Riconvalidazione di Geotrichum suaveolens (Lindn.) Cif. Atti Ist. Bot. Lab. Critt. Univ. Pavia Serie 5, 19, 3-7.

2. Diddens (H. A.) et Lodder (J.), 1942. - Die anaskosporogenen Hefen, II Hälfte, N. V. Noord-Hollandse Uit. Mg., Amsterdam.

3. Lodder (J.) et Kreger-VAN RiJ (N. J. W.), 1952. - The yeasts. A taxonomic study. North-Holland Publi. Cy, Amsterdam.

4. Morenz (J.), 1964. - Taxonomische Untersuchungen zur Gattung Geotrichum Link. Mykolog. Schriftenreihe, 2, 33-64.

5. SAËZ (H.), 1968. - Geotrichum gracile (Weigmann et Wolff 1909) Windisch 1952, un endosporé. Mykosen, II, 5, 347-352.

6. - , 1968. - Etude de quatre champignons arthrosporés formant des endospores. Microbiol. Espan., 21, 3-4, 193-204.

7. -, 1969. - Formation d'endospores chez Geotrichum candidum. Ann. Parasit. Hum. Comp., 44, 2, 197-204.

8. Walt VAn DeR (J. P.), 1959. - Endomyces reessii nov. spec. Antonie v. Leeuwenh., $25,458-464$.

9. Windisch (S.), 1951-1952. - Zur Biologie und Systematik des Milchschimmels und einiger ähnlicher Formen. I. Der Milchschimmel (Endomyces lactis) und Endomyces magnusii. II. Milchschimmelähnliche Pilze. Beiträge zur Biol. der Pflanz., 28, 2, 69-130 et $29,2,143-170$.

Nous sommes heureux d'exprimer notre reconnaissance aux Docteurs: M $^{\text {He }}$ Slooff, MM. Caretta, Van der Walt, Wickerham et aux Professeurs Morenz et Windisch pour les souches, documents et renseignements qu'ils ont eu l'amabilité de nous envoyer. 